\title{
Fluctuation-induced odd-frequency pairing
}

\author{
Odd-frequency spin-triplet instability in disordered electron liquid \\ Authors: Vladimir A. Zyuzin and Alexander M. Finkel'stein \\ arXiv:1912.04258
}

\section{Recommended with a Commentary by Jörg Schmalian, Karlsruhe Institute of Technology}

Cooper pairs are two-particle bound states. This gives rise to constraints for the Cooperpair wave function due to the Pauli principle, a behavior captured by the anomalous expectation value $F_{\alpha \beta}(\mathbf{k})=\left\langle c_{\mathbf{k} \alpha} c_{-\mathbf{k} \beta}\right\rangle$ with momentum $\mathbf{k}$ and spin projections $\alpha$, $\beta$. The anti-commutation of fermionic operators implies $F_{\beta \alpha}(-\mathbf{k})=-F_{\alpha \beta}(\mathbf{k})$. For systems with inversion symmetry, where $\mathbf{k} \rightarrow-\mathbf{k}$ is a symmetry operation, it follows that the minus sign is either due to an antisymmetry in spin space (spin singlet pairing) or in real space (spin triplet pairing).

In 1974 Berezhinskii[1] realized that one can consider a more general class of dynamic pairing correlation functions $F_{\alpha \beta}\left(\mathbf{k}, t-t^{\prime}\right)=\left\langle T c_{\mathbf{k} \alpha}(t) c_{-\mathbf{k} \beta}\left(t^{\prime}\right)\right\rangle$. He showed that the combined action of spin permutation, parity, and time permutation (not time reversal) gives rise to a minus sign. Thus, dynamic pairing correlations can be odd under the exchange of any of these variables and allow for a classification under time permutations:

$$
F_{\alpha \beta}^{(r)}(\mathbf{k}, \omega)= \pm F_{\alpha \beta}^{(a)}(\mathbf{k},-\omega)
$$

Here, $(r)$ and $(a)$ refer to the retarded and advanced version of the correlation function. The upper sign corresponds to the usual, even-frequency pairing. Odd frequency pairing, characterized by the lower sign, occurs for a state that is odd under time permutation[1]. For recent reviews on odd-frequency pairing, including a derivation of Eq.1, see Refs.[2, 3]. A careful analysis of the properties of Green's functions under time permutation in Refs. [4, 5] clarified the confusion that existed with regards to the thermodynamic stability of oddfrequency pairing. In addition, odd-frequency pairing was convincingly demonstrated to occur as a consequence of a proximity effect to states with broken time-reversal or translation symmetry $[6,7,8,9]$. Whether it can occur as a state of matter in a bulk system is a much discussed issue; see Refs.[3] for further references. Clearly, having a controlled approach that leads to a state with odd-frequency pairing is highly desirable.

Zyuzin and Finkel'stein (ZF) propose a promising approach to odd-frequency pairing in bulk systems. They consider interactions in a disordered conductor and show that the much sought-after odd-frequency pairing in the even-parity, triplet channel is likely to emerge via a fluctuation-induced instability. A crucial ingredient is the vicinity to an instability with ordinary, even-frequency, singlet, $s$-wave superconductivity. 


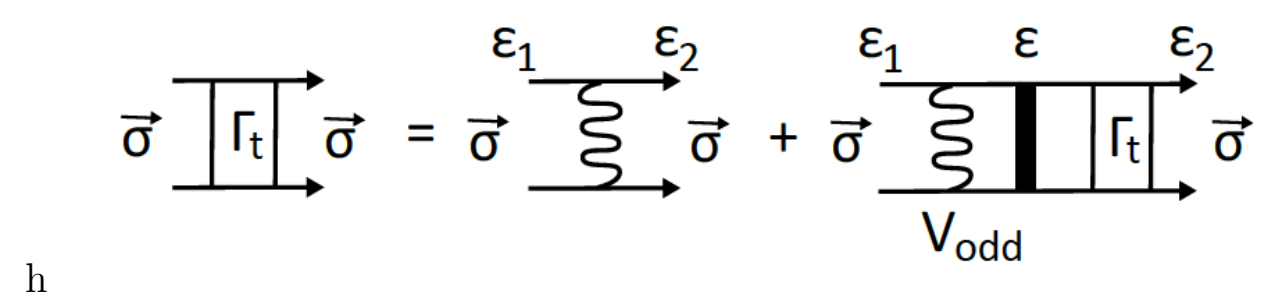

Figure 1: Effective interaction $\Gamma_{t}$ in the spin-triplet Cooper channel of a disordered metal. Here, the black box stands for a Cooperon mode. Only the odd component $V_{\text {odd }}\left(\epsilon_{1}, \epsilon_{2}\right)=$ $\frac{1}{4}\left(V_{\mathrm{t}}\left(\epsilon_{1}, \epsilon_{2}\right)-V_{\mathrm{t}}\left(-\epsilon_{1}, \epsilon_{2}\right)-V_{\mathrm{t}}\left(\epsilon_{1},-\epsilon_{2}\right)+V_{\mathrm{t}}\left(-\epsilon_{1},-\epsilon_{2}\right)\right)$ is able to induce triplet pairing. From Zyuzin and Finkel'stein, arXiv:1912.04258

ZF start their analysis using the non-linear $\sigma$-model formulation of disordered interacting electrons and analyze interference effects between interactions in the particle-hole and particle-particle channel. In the usual fashion, the formalism includes soft diffuson and Cooperon modes as they occur in diffusive metals. The small parameter of the formalism is the resistance per square, $\rho$, measured in units of $h / e^{2}$. The first insight, indicated in Fig.1, is that an analysis of the relevant ladder diagrams in the spin-triplet Cooper channel $\Gamma_{t}$ is sensitive only to the odd-in-frequency bare contribution $V_{\text {odd }}$ of this channel. Only this contribution can induce triplet pairing, which then occurs in the odd-frequency channel.

In a second part of their analysis, ZF identify a specific microscopic mechanism that gives rise to a sizable contribution to $V_{\text {odd }}\left(\epsilon_{1}, \epsilon_{2}\right)$. They find that the interference between interactions in the even-frequency s-wave pairing channel and a spin-density particle-hole interaction gives the dominant contribution. Thus, if one is in a regime where at least one of the two interactions is large, it offsets the smallness of the underlying expansion parameter $\rho$, and odd-frequency pairing becomes a possibility.

A numerical analysis of $V_{\text {odd }}\left(\epsilon_{1}, \epsilon_{2}\right)$ due to this mechanism is shown in Fig.2. ZF find a sizable contribution, of order unity in the appropriate units. They stress that in their findings the singular dependence $V_{\text {odd }}\left(\epsilon_{1}, \epsilon_{2}\right) \sim \operatorname{sign}\left(\epsilon_{2}\right)$ on $\epsilon_{2}$ does not rely on the fact that $\epsilon_{1}$ must be smaller than $\epsilon_{2}$, and vice versa. This surprising finding is in contrast to the behavior with leading logarithmic divergencies summed up within a renormalization group program. High and low-energy degrees of freedom are coupled in a fashion that is more reminiscent of, e.g. Landau damping. The results demonstrate that the rich spectrum of soft excitations in disordered conductors allows for new mechanisms of pairing instabilities.

In order to suppress $s$-wave pairing, ZF further consider a finite magnetic field. Within a lowest-Landau-level analysis they show that the odd-frequency pairing instability occurs for magnetic fields significantly above $H_{c 2}$, up to $H \approx 2.5 H_{c 2}$. For even larger fields the oddfrequency pairing instability is suppressed as well. Finally, ZF argue that their finding offers an explanation for i) the observation of Refs.[10, 11] of single-particle gaps in disordered superconductors that were interpreted in terms of preformed even-frequency pairs and ii) the observation of field-induced superconductor-insulator transitions in such systems[12].

The most peculiar finding of Zyuzin and Finkel'stein is the highly singular odd-frequency pairing amplitude that emerges from the interference of even-frequency pairing and spindensity interactions in a disordered metal. It is fair to say that we do not yet fully understand 


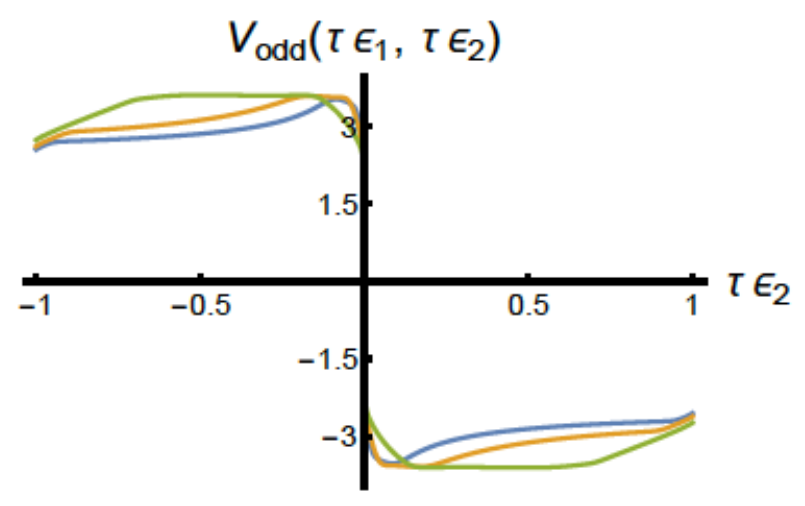

Figure 2: Odd-frequency projected interaction $V_{\text {odd }}$ resulting from the interference of the singlet Cooper pairing interaction $\Gamma_{c}$ and the particle-hole spin-density interaction $\Gamma_{2}$ as function of $\epsilon_{2}$. $V_{\text {odd }}$ is given in units of $\rho\left|\Gamma_{c}\right| \Gamma_{2}$ for $\tau \epsilon_{1}=0.05$ (blue) $\tau \epsilon_{1}=0.1$ (orange), and $\tau \epsilon_{1}=0.3$ (green) at low temperature $T=0.01 \tau^{-1} . \tau$ is the impurity scattering time and $\rho$ is the resistance per square measured in units of $h / e^{2}$. From Zyuzin and Finkel'stein, arXiv:1912.04258

this numerical result, shown in Fig.2. A fluctuation-induced pairing instability emerges in a fashion that can not be efficiently captured within the usual renormalization group language. It is also unclear whether the newly identified pairing instability gives rise to a true transition to a superconducting phase. ZF suspect that it doesn't. This is important for their interpretation of the above-mentioned experiments. The good news are that the approach used by ZF allows, at least in principle, checking whether there are fluctuations that destroy phase-coherent, odd-frequency superconductivity. Either way, this manuscript offers a new approach towards odd-frequency pairing in bulk systems with highly unusual results. It provides an example for fluctuation-induced superconductivity that cannot be rationalized within a scheme of energy hierarchies, such as in the parquet renormalization group program.

\section{References}

[1] V. L. Berezinskii, Pis'ma Zh. Eksp. Teor. Fiz 20, 628-631 (1974).

[2] Y. V. Fominov, Y. Tanaka, Y. Asano, M. Eschrig, Odd-frequency superconducting states with different types of Meissner response: Problem of coexistence, Phys. Rev. B 91 144514 (2015).

[3] J. Linder and A. V. Balatsky, Rev. Mod. Phys. 91, 045005 (2019).

[4] D. Belitz, and T. R. Kirkpatrick, Phys. Rev. B 60, 3485-3498 (1999).

[5] D. Solenov, I. Martin, and D. Mozyrsky, Phys. Rev. B 79, 132502 (2009). 
[6] F. S. Bergeret, A. F. Volkov, and K. B. Efetov (2004), Phys. Rev. Lett. 86, 4096-4099 (2001).

[7] A. F. Volkov, F. S. Bergeret, and K. B. Efetov, Phys. Rev. Lett. 90, 117006 (2003).

[8] M. Eschrig, J. Kopu, J. C. Cuevas, and G. Schön, Phys. Rev. Lett. 90, 137003 (2003).

[9] A. I. Buzdin, Rev. Mod. Phys. 77, 935-976 (2005).

[10] T. Dubouchet, B. Sacepe, J. Seidemann, D. Shahar, M. Sanquer, C. Chapelier, Nature Physics 15, 288 (2019).

[11] B. Sacepe,T. Dubouchet, C. Chapelier, M. Sanquer, M. Ovadia, D. Shahar, Nature Physics 7, 239-244 (2011).

[12] V. F. Gantmakher, M. V. Golubkov, V. T. Dolgopolov, A. Shashkin, G. E. Tsydynzhapov, JETP Lett. 71, 473-476 (2000); G. Sambandamurthy, L. W. Engel, A. Johansson, D. Shahar, Phys. Rev. Lett. 92, 107005 (2004); M. A. Steiner, G. Boebinger, A. Kapitulnik, Phys. Rev. Lett. 94, 107008 (2005); T. I. Baturina, C. Strunk, M. R. Baklanov, A. Satta, Phys. Rev. Lett. 98, 127003 (2007). 